\title{
Radon Transform based Automatic Metal Artefacts Generation for 3D Threat Image Projection
}

\author{
Najla Megherbi, Toby P. Breckon, Greg T. Flitton, Andre Mouton \\ School of Engineering, Cranfield University, Bedfordshire, UK \\ n.megherbi, toby.breckon, g.t.flitton, a.mouton@cranfield.ac.uk
}

\begin{abstract}
Threat Image Projection (TIP) plays an important role in aviation security. In order to evaluate human security screeners in determining threats, TIP systems project images of realistic threat items into the images of the passenger baggage being scanned. In this proof of concept paper, we propose a 3D TIP method which can be integrated within new 3D Computed Tomography $(\mathrm{CT})$ screening systems. In order to make the threat items appear as if they were genuinely located in the scanned bag, appropriate CT metal artefacts are generated in the resulting TIP images according to the scan orientation, the passenger bag content and the material of the inserted threat items. This process is performed in the projection domain using a novel methodology based on the Radon Transform. The obtained results using challenging 3D CT baggage images are very promising in terms of plausibility and realism.
\end{abstract}

\section{INTRODUCTION}

It is well known that detecting threat items using human screeners is important for aviation security. To enhance and monitor security screener detection performance, Threat Image Projection (TIP) technology is used. This is incorporated in the X-ray screening machines to assess aviation security screeners in detecting threats during routine baggage screening operations. In cabin baggage screening, X-ray images of realistic threat items are projected by the TIP system into the X-ray images of the passenger bags being scanned $[1,2]$. The use of TIP technology is currently limited to the conventional 2D X-ray baggage screening systems in which the screener looks at the 2D X-ray image of the bag being scanned $[3,4]$.

CT technology initially developed for 3D medical imaging applications is in increasing use in airports for luggage inspection [5]. This is complemented by a parallel work in automatic threat object detection [6-11], noise reduction [12], and Metal Artefacts Reduction (MAR) [13-15] in such imagery. Clearly, extending the use of TIP technology in the $3 \mathrm{D}$ volumetric CT scanning systems would be of great importance for enhancing screener threat detection performance. Instead

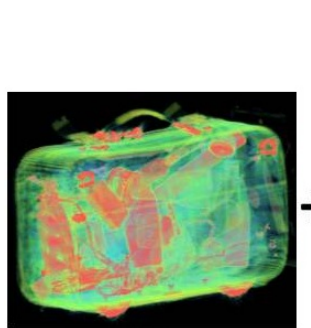

(a)

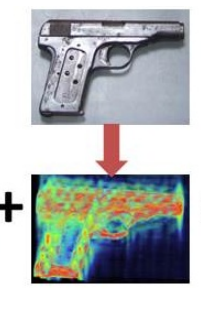

(b)

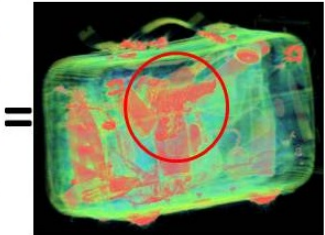

(c)
Figure 1. 3D TIP system: (a) 3D CT image of a real and harmless passenger bag, (b) Threat item 3D CT image, (c) 3D TIP image

of superimposing 2D images of threat items into the $2 \mathrm{D}$ $\mathrm{X}$-ray images of passenger bags, 3D TIP requires the 3D insertion of threat item images into the 3D CT image of the passenger bag as depicted in Figure 1. To fulfil this task effectively, it is necessary to insert the threat item in an appropriate place in the passenger bag without violating the existing baggage contents, for example cutting other objects.

In addition, as it is well known, the use of CT technology in scanning objects creates imaging artefacts in the reconstructed 2D and 3D images [16]. Unlike medical imagery where CT artefacts have several causes [16], CT artefacts in baggage imagery are mainly due to the presence of the metal [15]. In practice, most scanned bags contain metal (zips, buckles...). Since most threat items are made from metal, their insertion within the baggage CT scans makes the metal artefacts more complex as this increases the amount of metal in the scanned bag. In order to make the 3D TIP realistic, it is necessary to generate CT metal artefacts in the resulting 3D TIP images.

In prior work, only one attempt has been made to integrate 3D TIP within 3D CT scanning systems [17]. In this work, the original CT metal artefacts produced by the threat item from its original scan are added to the resulting 3D TIP regardless of the bag content. In practice, this is not valid as the production of CT metal artefacts depends on the scan orientation of the object, the material of the object, and the bag content. Additionally, this provides perceptible visual cues to human 
screeners as the artefacts originating from the inserted threat item would be inconsistent with the original bag artefacts.

In this research work, in order to make the inserted threat both realistic and plausible with reference to the existing baggage contents, a novel method inspired from Metal Artefact Reduction (MAR) techniques in the medical field is utilised $[15,18,19]$. Based on the passenger bag content and the material of the inserted threat item, appropriate CT metal artefacts are automatically generated in the projection data using a novel approach centred on the use of the Radon transform [20].

\section{CT METAL ARTEFACTS}

The problem of metal artefacts in X-ray CT scanning is well studied in medical imaging applications $[15,18$, 19, 21, 22]. Metal artefacts are caused by the presence of high density objects in the scan field of view. The origin of metal artefacts has been studied extensively in the literature and several assumptions have been made.

The primary assumption is that as metal objects are high-attenuation objects (with a high atomic number) they heavily attenuate the X-ray beams. As a result relatively fewer photons reach the scanner detectors when passing through metal objects (photon starvation effect) resulting in corrupt or missed data in the measured projection data (sinogram). Subsequently, during the CT image reconstruction process, the filtered back projection (FBP) algorithm (the standard method of CT image reconstruction) amplifies the missing data in the sinogram due to the logarithmic function involved and leads to artefacts in the reconstructed $\mathrm{CT}$ images $[18,19]$.

A secondary assumption is that all artefacts, including metal artefacts, result from the discrepancies between the actual non-linear acquisition process and the ideal mathematical model used in the reconstruction process. According to this assumption, CT artefacts are due to system non-linearities which are not included in the reconstruction model. These non-linearities include beam hardening effect, partial volume effect, scatter effect, exponential edge-gradient effect, beam starvation effect and noise. This imperfection (or lack) in the modelling of these non-linear effects result in inconsistent measured projection data [21, 22].

Regardless the origin of metal artefacts, the effects of these artefacts in the reconstructed CT images is the same. Metal artefacts appear as dark and white streaks radiating from the metal objects and spreading across

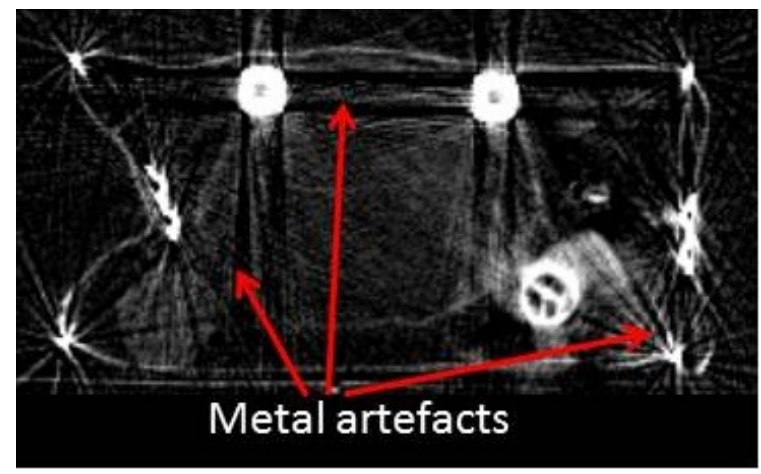

Figure 2. CT Metal artefacts in a CT slice from a cluttered baggage item

the whole reconstructed CT images. They are more prominent near to the metal objects and are function of the scan orientation of the bag and the material of the bag content (see Figure 2).

In Figures 3 and 4 we show an example of a scanned bag containing a gun placed in different orientations. As can be seen, the generated artefacts in both scans are different. This is due to the fact that the size of the gun metal part that the X-rays have intersected is not the same because of the different orientation. Figure 3 and 4 demonstrate thus that the intensity of the metal artefacts and their direction depend on the direction of the scanned objects (bag).

In addition, metal artefacts depend on the nature of the bag content (see Figure 5). In particular the material of the objects located in the shadow of the metal object affects the intensity of the metal artefacts and their direction. The high CT density these objects have the strong metal artefacts. In extreme case when several metallic objects are present, pronounced metal artefacts are produced in the reconstructed CT image as depicted in Figure 6.

\section{METAL ARTEFACTS GENERATION (MAG) USING RADON TRANSFORM}

In our TIP methodology we are processing threat items mostly made from metal. From the earlier discussion, it is necessary to take into consideration metal artefacts in the TIP process in order to make the threat items appear as if they were genuinely located in the scanned bag. Our proposed MAG procedure depicted in Figure 7 is inspired from the established MAR projectionreplacement techniques in medical imaging applications $[18,19]$. In a similar vein to these methods, our Metal Artefact Generation (MAG) procedure starts first by mapping the original 3D CT image of the clear bag 

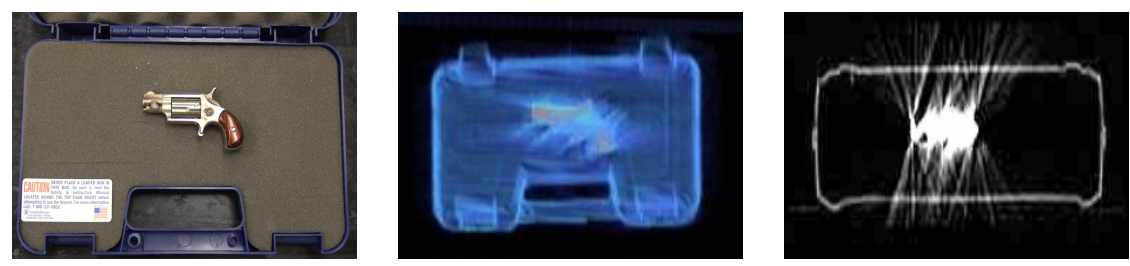

Figure 3. Metal artefacts are function of the scan orientation of the objects. Left to right: picture of the scanned test $\mathrm{bag} /$ case interior (gun horizontal), generated CT volume, CT slice.
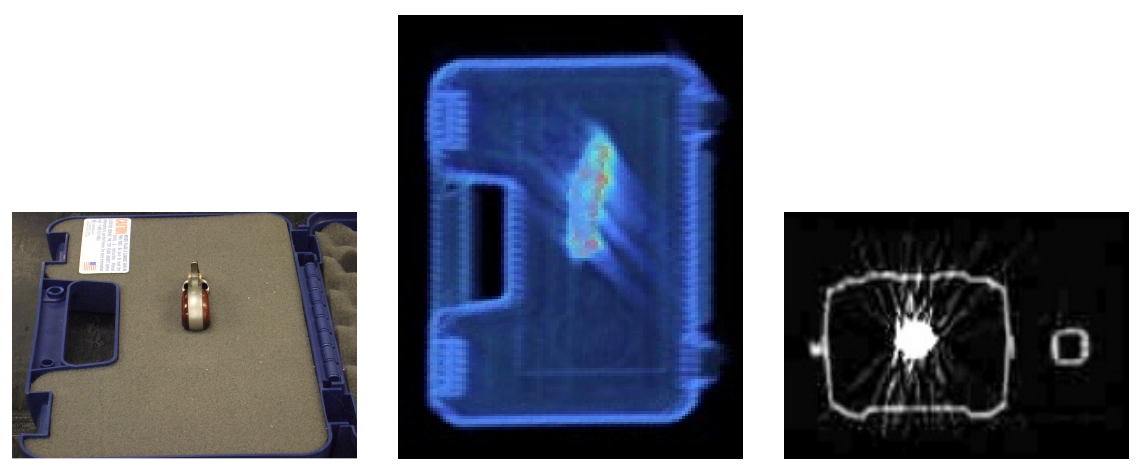

Figure 4. Metal artefacts are function of the scan orientation of the objects. Left to right: picture of the scanned test $\mathrm{bag} /$ case interior (gun vertical), generated CT volume, CT slice.
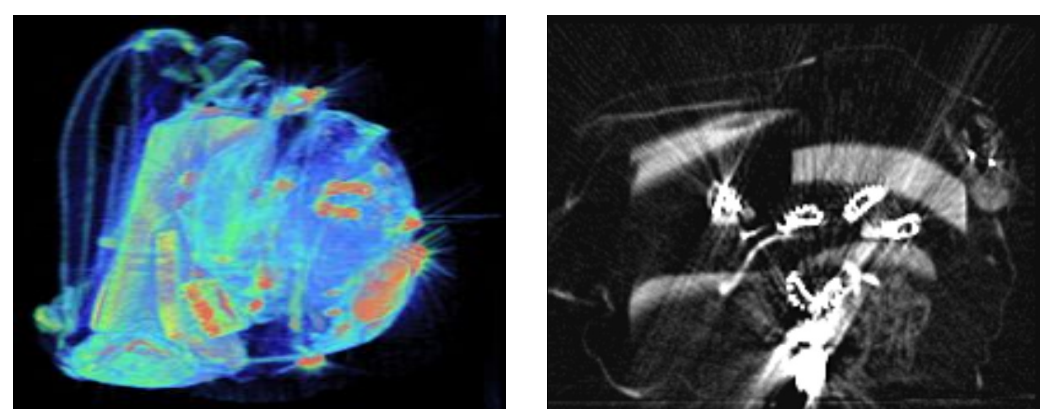

Figure 5. Metal artefacts are function of the material of the bag content. Left: 3D CT image, right: CT slice
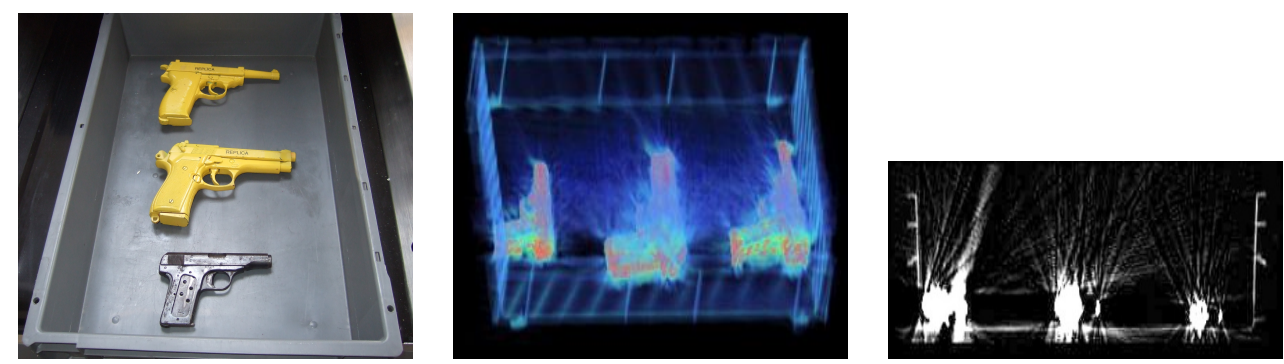

Figure 6. Metal artefacts in the presence of several metallic objects. Left to right: picture of the scanned bag, generated CT volume, CT slice. 
(i.e. harmless passenger bag) its metal-only 3D CT image and the 3D CT metal-only image of the (artefact free) threat item to the projection domain via Radon transform [20]. The output of this step is images known as sinograms. In order to mimic the CT scanner used during the real scans, the CT scanner geometry and the projection parameters used to compute the Radon transform are defined according to the actual CT scanner geometry. This enables our method to include the scan orientation of the bag in the MAG process. The artefact free threat item 3D CT image is obtained first by isolating the 3D threat item from its surroundings in the threat CT image and then applying a suitable CT density thresholding to remove the artefacts and the noise.

The metal-only images of the clear bag and the artefact free threat item are obtained by segmenting the metal objects in their original CT image by thresholding using a suitable metal CT density threshold. This step exploits the fact that metal objects in CT images have high CT value compared to other objects. Subsequently the metal traces corresponding to the metal objects of the clear bag and the metal part of the artefact free threat item are combined in one projection image. A mask corresponding to the whole metal traces is marked in the sinogram of the clear bag CT image.

In conventional MAR projection-replacement based methods [15], this mask corresponds to the corrupted area in which projection bins are affected by metallic objects and which need to be replaced by surrogate data. Marking this corrupted area in the Radon domain is equivalent to determining in the 3D CT TIP image (clear bag with the threat item) all rays passing through the metallic objects originating from the bag and threat item. CT metal artefacts emerging from the metallic objects are spread across these lines.

In order to generate metal artefacts in the clear bag CT image, the projection bins in the marked mask in the clear bag sinogram are thus made inconsistent with their neighbourhood unlike MAR projectionreplacement based methods in which the projection bins in this mask are replaced by interpolated data.

The underlying idea behind this is to mimic a real CT scanning of a metal object by making the sinogram values corrupted and inconsistent with their neighbourhood if the corresponding X-rays have intersected the metal object. In fact, since metal objects are highattenuation objects, they heavily attenuate the X-ray beams and consequently only few photons reach the scanner detectors. This effect known as photon starvation effect produces indeed corrupted data in the sino- gram and gives rise to artefacts in the reconstructed 2D and $3 \mathrm{D}$ images.

In order to corrupt the projection bins of the marked mask in the clear bag sinogram, we have used an empirical function. As we will show shortly, by following the above steps, consistent metal artefacts are generated within the bag CT images which are a function of the scan orientation of the bag, the material of the bag content and the material of the inserted threat item. As depicted in Figure 7, once the metal artefacts are generated in the Radon space, the resulting modified sinogram is reprojected into the CT domain. The resulting reconstructed $\mathrm{CT}$ image corresponds to the original clear bag CT image corrupted by metal artefacts originating from the threat item metal part and the clear bag metal objects. The final 3D TIP image is obtained by combining the resulting $\mathrm{CT}$ image with the artefact free threat item CT image (e.g. Figure 7).

In this work the location in the $3 \mathrm{D}$ clear bag $\mathrm{CT}$ image into which a threat item 3D CT image is inserted is determined manually and this automatic placement is an area of current work.

\section{EXPERIMENTAL RESULTS}

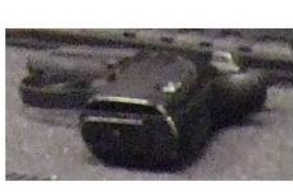

(a)

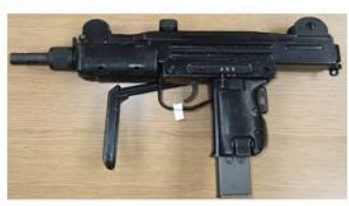

(b)
Figure 8. Examples of threat items used in the experiments

Since in our work we are interested in metal artefacts generation in the TIP images we use guns as threat items as they are mostly made from metal. In Figure 8 we show two example guns used for this experiment. In addition, in order to demonstrate our method we select challenging 3D CT baggage images that reflect real passenger bags.

In Figure 9 and 10, we show the results of our approach. As it is difficult to see the artifacts in the 3D CT images, we have selected two representative slices for each case which highlights the performance of our method.

As the examples of Figures 9 and 10 illustrate, the threat items look as if they were real objects of within the scanned bags. The generated CT artefacts using our method are consistent with those of the passenger bag. The whole 3D CT images of the bags are 


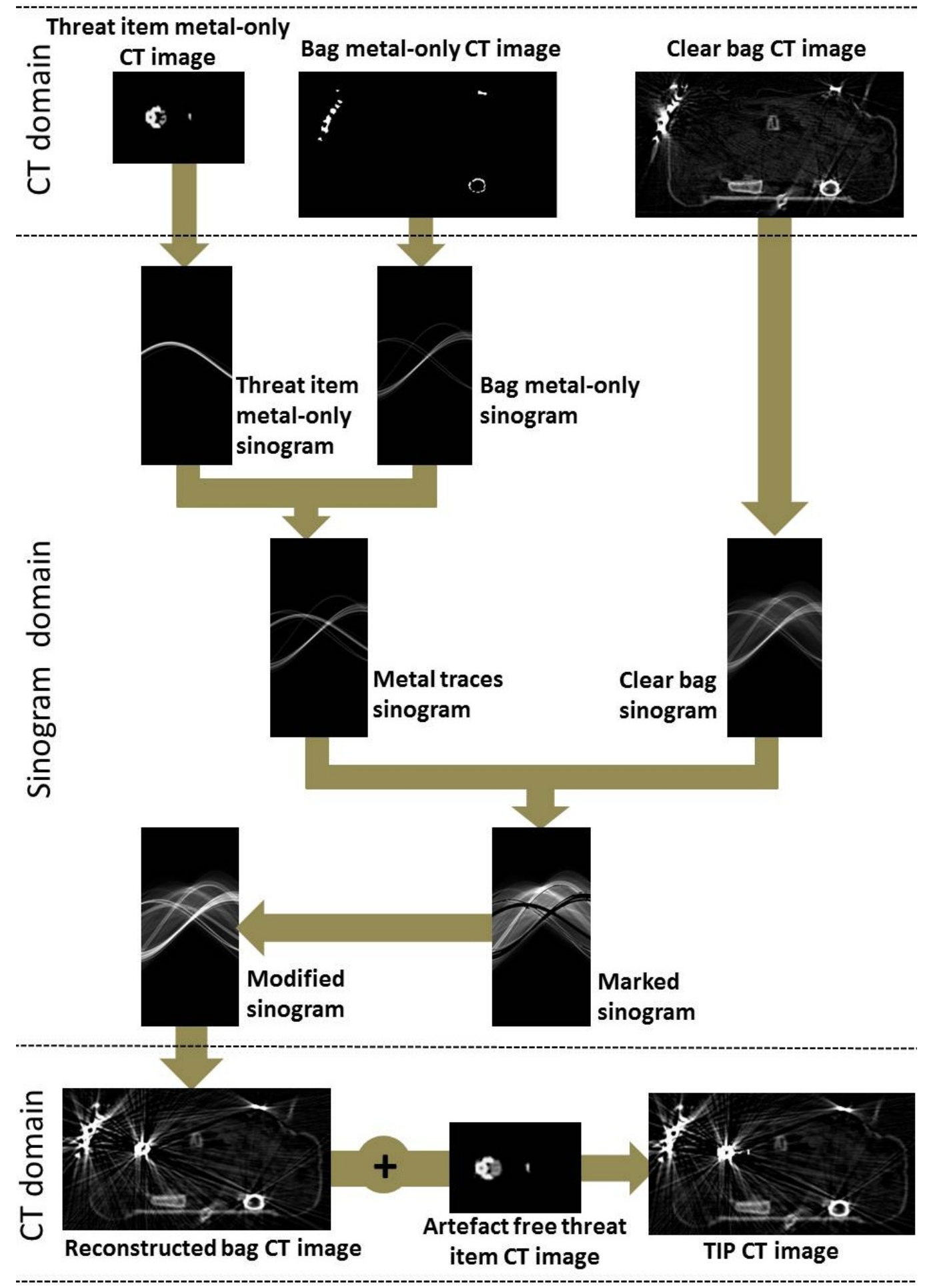

Figure 7. Flow chart of our MAG method depicted using 2D CT slices 

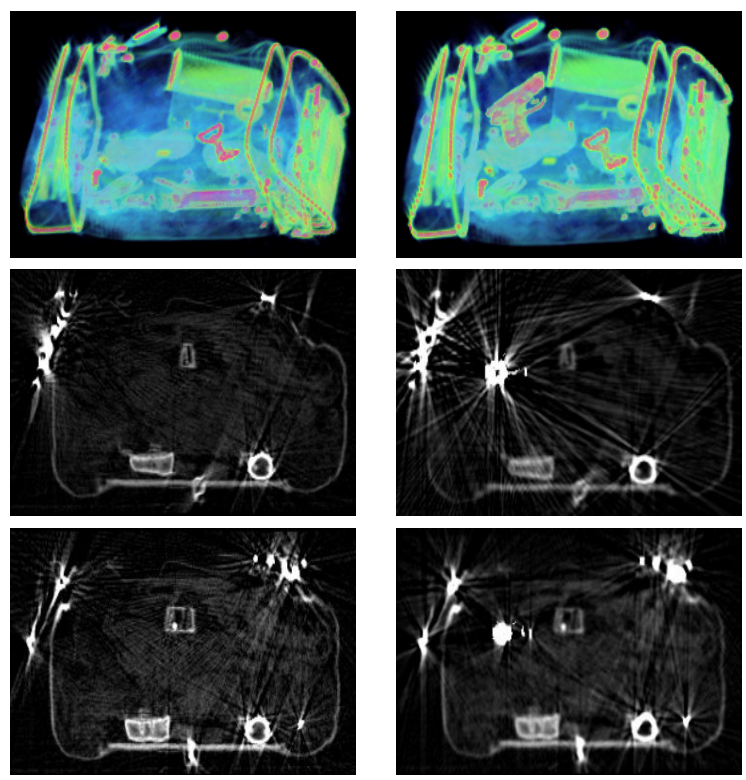

Figure 9. Insertion of gun(a). Left: original CT images, right: TIP images. Top: 3D CT images, bottom: CT slices

corrupted by streaking artefacts originating from the threat items and the bags metal objects. Note in particular the strong streaking which takes place in regions near to the metallic objects.

Similarly to MAR methods developed in medical applications it is difficult to evaluate our method qualitatively since we are using real data. Nonetheless, as the TIP systems are used by the aviation security screeners in their task of passenger bags visual inspection, we have asked three experts in CT baggage data to look carefully at our 3D CT TIP images and evaluate the performance. None of these experts was able to determine that the threat items are not part of the original scanned bags and that they have been artificially inserted inside these bags under blind test conditions.

\section{CONCLUSION}

Enhancing threat detection performance of airport screeners is important for aviation security. While 3D CT screening systems are widely used in airports, current TIP systems used to assess aviation screeners in detecting threats are only designed for 2D X-ray screening machines. In this paper we propose a 3D TIP method designed for 3D CT scanner machines. As scanning objects using $\mathrm{CT}$ technology leads to imaging artefacts in the reconstructed 3D CT images, it is necessary to generate appropriate $\mathrm{CT}$ artefacts in the resulting $3 \mathrm{D}$ TIP images in order to make the threat items appear both plausible and realistic. As metal artefacts are the
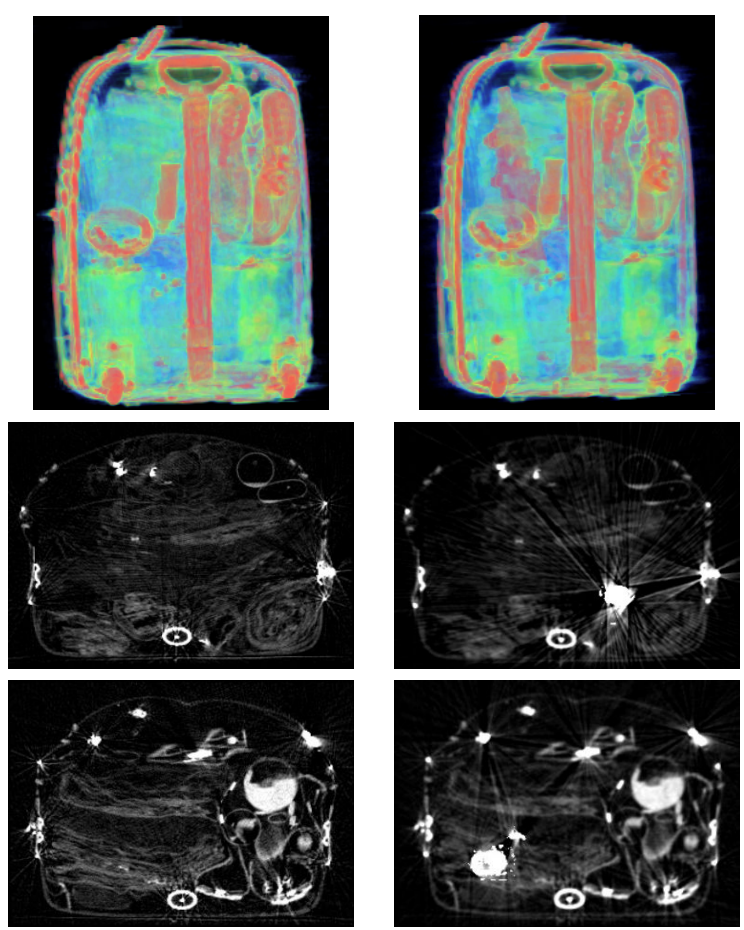

Figure 10. Insertion of gun(b). Left: original CT images, right: TIP images. Top: 3D CT images, bottom: CT slices

most prominent artefact encountered in $\mathrm{CT}$ baggage data, we have proposed a method inspired for the wellestablished MAR projection-replacement techniques in medical imaging applications to generate metal artefacts in the resulting 3D TIP images. Depending on the scan orientation, bag contents and the material of the threat item, appropriate metal CT artefacts are automatically generated consistently within the bag CT image. The metal artefacts generation is performed in the projection domain using Radon transform. Our new method has been tested on challenging 3D CT baggage images and real threat items. The results obtained are very promising and are qualitatively evaluated by a panel of human experts.

The insertion location into which a threat 3D image is inserted is currently determined manually. Automatic insertion location determination for currently under investigation in future work.

This project was funded under the Innovative Research Call in Explosives and Weapons Detection 2010 initiative. This is a Cross-Government programme sponsored by a number of Departments and Agencies under the UK Government's CONTEST strategy in partnership with the US Department of Homeland Security. 


\section{REFERENCES}

1. K. Catchpole, J. Fletcher, A. McClumpha, A. Miles, and A. Zar, "Threat image projection: applied signal detection for aviation security," Engineering Psychology and Cognitive Ergonomics, vol. 6, no. 1, pp. 231-237, 2001.

2. V. Cutler and S. Paddock, "Use of threat image projection (TIP) to enhance security performance," 43rd Annual Int. Carnahan Conf. in Security Technology, pp. 46-51, 2009.

3. S. Steiner-Koller, A. Bolfing, and A. Schwaninger, "Assessment of X-ray image interpretation competency of aviation security screeners," 43rd Annual Int. Carnahan Conf. on Security Technology, pp. 20-27, 2009.

4. F. Hofer and A. Schwaninger, "Using threat image projection data for assessing individual screener performance," WIT Transactions on the Built Environment, vol. 82, pp. 417-426, 2005.

5. S. Singh and M. Singh, "Explosives detection systems (EDS) for aviation security," Signal Processing, vol. 83, no. 1, pp. 31-55, 2003.

6. N. Megherbi, J. Han, G. Flitton, and T. Breckon, "A comparison of classification approaches for threat detection in CT based baggage screening," in Proc. International Conference on Image Processing, pp. 3109-3112, IEEE, September 2012.

7. N. Megherbi, G. Flitton, and T. Breckon, "A classifier based approach for the detection of potential threats in CT based baggage screening," in Proc. International Conference on Image Processing, pp. 1833-1836, IEEE, September 2010.

8. G. Flitton, T. Breckon, and N. Megherbi, "A comparison of 3D interest point descriptors with application to airport baggage object detection in complex CT imagery," Pattern Recognition, vol. 46, pp. 2420-2436, September 2013.

9. G. Flitton, T. Breckon, and N. Megherbi, "Object recognition using 3D SIFT in complex CT volumes," in Proc. British Machine Vision Conference, pp. 11.1-12, September 2010.

10. G. Flitton, T. Breckon, and N. Megherbi, "A 3D extension to cortex like mechanisms for 3D object class recognition," in Proc. International Conference on Computer Vision and Pattern Recognition, pp. 3634-3641, IEEE, June 2012.

11. N. Megherbi, T. Breckon, and G. Flitton, "Investigating existing medical CT segmentation techniques within automated baggage and package inspection," in Proc. SPIE Security and Defence: Optics and Photonics for Counterterrorism, Crime Fighting and Defence, SPIE, 2013. to appear.
12. A. Mouton, G. Flitton, S. Bizot, N. Megherbi, and T. Breckon, "An evaluation of CT image denoising techniques applied to baggage imagery screening," in Proc. International Conference on Industrial Technology, pp. 1063-1068, IEEE, February 2013.

13. A. Mouton, N. Megherbi, G. Flitton, S. Bizot, and T. Breckon, "A novel intensity limiting approach to metal artefact reduction in 3D CT baggage imagery," in Proc. International Conference on Image Processing, pp. 2057-2060, IEEE, September 2012.

14. A. Mouton, N. Megherbi, T. Breckon, K. Van Slambrouck, and J. Nuyts, "A distance weighted method for metal artefact reduction in CT," in Proc. International Conference on Image Processing, IEEE, September 2013. to appear.

15. A. Mouton, N. Megherbi, K. Van Slambrouck, J. Nuyts, and T. Breckon, "An experimental survey of metal artefact reduction in computed tomography," Journal of X-Ray Science and Technology, vol. 21, pp. 193-226, May 2013.

16. J. Barrett and N. Keat, "Artifacts in CT: Recognition and avoidance," RadioGraphics, vol. 24, pp. 1679 - 1691, 2004.

17. Y. O. Yildiz, D. Q. Abraham, S. Agaian, and K. Panetta, "3D threat image projection," Proc. SPIE, vol. 6805, 2008.

18. A. Mehranian, M. Ay, A. Rahmim, and H. Zaidi, "Sparsity constrained sinogram inpainting for metal artifact reduction in X-ray computed tomography," IEEE Nuclear Science Symposium and Medical Imaging Conference, Barcelona, Spain, pp. 3694-3699, 2011.

19. A. Mehranian, M. Ay, A. Rahmim, and H. Zaidi, "Metal artifact reduction in CT-based attenuation correction of PET using sobolev sinogram restoration," IEEE Nuclear Science Symposium and Medical Imaging Conference, Barcelona, Spain, pp. 2936-2942, 2011.

20. S. Deans, "The radon transform and some of its applications," New York: John Wiley \& Sons, 1983.

21. B. D. Man, J. Nuyts, P. Dupont, G. Marchal, and P. Suetens, "Metal streak artifacts in X-ray computed tomography: a simulation study," IEEE Transactions on Nuclear Science, vol. 46, no. 3, pp. $691-696,1999$.

22. J. Williamson, B. Whiting, J. Benac, R. Murphy, G. Blaine, J. O'Sullivan, D. Politte, and D. Snyder, "Prospects for quantitative computed tomography imaging in the presence of foreign metal bodies using statistical image reconstruction," Med Phys., vol. 29, no. 10, pp. 2404-2418, 2002. 